\title{
Infarto perioperatorio y cirugía no cardíaca
}

\author{
Perioperative infarction and non-cardiac surgery
}

María Alejandra Cisneros ${ }^{1}$ John Jader Núñez Enríquez², Andrés Fernando Bolaños Delgado³, Cristian Felipe Correa Gallego², Juan Felipe Vidal Martínez², Erwin Giovanny González Hoyos ${ }^{4}$

\begin{abstract}
Introduction: Perioperative cardiovascular events occur in up to $5-8 \%$ of all patients older than 45 years undergoing non-cardiac surgery. Objective: The objective of this article is to perform an updated narrative review about perioperative infarction that occurs in the context of non-cardiac surgery. Methods: A search was proposed with different combinations of the terms: Myocardial infarction (MeSH), perioperative period (Majr), postoperative period (Majr) and anesthesia (MeSH), in PubMed (Medline), OVID y EMBASE databases. Results: Around 29 articles were found with important information for the present review. Conclusions: Myocardial injury, as well as perioperative infarction are common entities, but difficult to diagnose. Its identification and prompt treatment generate a great impact on morbidity and mortality, which is why its active search should be carried out, especially in patients with high cardiovascular risk.
\end{abstract}

\section{RESUMEN}

Introducción: Los eventos cardiovasculares perioperatorios ocurren hasta en un 5\%-8\% de todos los pacientes mayores de 45 años sometidos a cirugía no cardíaca. Objetivo: El objetivo del presente artículo es realizar una revisión narrativa actualizada acerca del infarto perioperatorio que ocurre en el contexto de la cirugía no cardíaca. Metodología: Se planteó una búsqueda con distintas combinaciones de los términos: Myocardial infarction (MeSH), perioperative period (Majr), postoperative period (Majr), anesthesia (MeSH), en las bases de datos PubMed (Medline), OVID y EMBASE. Resultados: Se encontraron alre-

\section{Key words:}

Myocardial infarction, perioperative period, postoperative period and anesthesia (MeSH)

\section{Palabras clave:}

Infarto de miocardio, período perioperatorio, período posoperatorio, anestesia (DeCS)

\footnotetext{
Médica y cirujana. Medilaser IPS. Florencia, Colombia.

Médico y cirujano. Hospital Universitario San José. Popayán, Colombia.

Médico y cirujano. Emermédica IPS. Medellín, Colombia.

Médico y cirujano. Hospital Susana López de Valencia. Popayán, Colombia.
}

Fecha de recepción: 11 de agosto de 2019

Fecha de aceptación: 22 de septiembre de 2019

\section{ORCID}

https://orcid.org/0000-0002-5716-1679

\section{Correspondencia:}

María Alejandra Cisneros

melejacisnerosc@gmail.com. 
dedor de 29 artículos con información importante para la realizar la presente revisión. Conclusiones: La lesión miocárdica, así como el infarto perioperatorios son entidades frecuentes, pero de difícil diagnóstico. Su identificación y pronto tratamiento genera un gran impacto sobre la morbimortalidad, por lo que debe realizarse su búsqueda activa, especialmente en pacientes con riesgo cardiovascular elevado.

\section{Introducción}

os eventos cardiovasculares perioperatorios ocurren hasta en un 5\%-8\% de todos los pacientes mayores de 45 años sometidos a cirugía no cardíaca. Estos incluyen el infarto agudo de miocardio, las arritmias ventriculares, la insuficiencia cardíaca y la muerte súbita[1],[2]. Por otro lado, una importante proporción de pacientes sufre aumento de biomarcadores de isquemia miocárdica, sin que desarrollen clínica ni cambios electromecánicos, considerándose pacientes que sufren lesión miocárdica, sin llegar a tener necrosis tisular[3]. El objetivo del presente artículo, es realizar una revisión narrativa actualizada acerca del infarto perioperatorio y la lesión miocárdica que ocurren en el contexto de la cirugía no cardíaca.

\section{Métodos}

Se realizó una búsqueda estructurada de la literatura con los términos: Myocardial infarction (MeSH), perioperative period (MeSH, Majr), postoperative period (MeSH, Majr), anesthesia (MeSH), en las bases de datos: PubMed (Medline), OVID y EMBASE; con las siguientes limitantes: artículos publicados en los últimos diez años, en idioma inglés o español, con texto completo disponible, reportando estudios realizados exclusivamente con humanos. Se incluyeron revisiones sistemáticas, ensayos clínicos aleatorizados en favor de determinar la existencia de directrices de tratamiento; así como estudios de cohorte o casos y controles para abordar otros tópicos como epidemiología y pronóstico. Por regla general, se excluyeron manuscritos editoriales, cartas al editor, estudios de corte transversal y artículos de revisión. Se presenta una síntesis de artículos que, a juicio de los autores, representa una adecuada evidencia para el estudio de la lesión miocárdica y el infarto perioperatorios.

\section{Resultados}

Mediante búsqueda estructurada, se identificaron alrededor de 29 manuscritos con información relevante para el desarrollo de la presente revisión. El flujograma de selección se describe en la Figura 1.

\section{Lesión miocárdica e infarto agudo de miocardio}

La lesión miocárdica por cirugía no cardíaca ( $\mathrm{LMCnC}$ ) corresponde a la elevación de biomarcadores cardíacos, la cual puede ocurrir hasta 30 días después del procedimiento quirúrgico y que puede estar asociada o no a síntomas. Su espectro incluye tanto pacientes que cumplen, como a aquellos que escapan a la definición universal de infarto, siempre en ausencia de condiciones que expliquen un alza no isquémica de los valores de troponina, incluyendo sepsis, tromboembolismo pulmonar, insuficiencia renal aguda y el estado postcardioversión[1],[4]. Mediante diversos estudios se ha determinado que, hasta el $85 \%$ de las elevaciones de troponina en el escenario perioperatorio, son de origen isquémico; y al menos el $20 \%$ de las LMCnC corresponde a un infarto agudo de miocardio (IAM), por lo que cualquier aumento debe estudiarse como si se tratara de un evento coronario[5]-[7].

\section{Etiología y fisiopatología}

Usualmente, la mayoría de los casos de infarto perioperatorio pueden clasificarse como tipo I o II, casi que en iguales proporciones. El tipo I, debido a la rotura de una placa aterotrombótica, se ha documentado hasta en 13\%-50\% los casos de AIM perioperatorio según distintas series. De cualquier modo, hasta 3 de cada 5 pacientes presentan lesiones fibroateromatosas que predisponen al disbalance entre la demanda y la oferta de oxígeno, que se exacerba bajo anestesia por hipotensión, hipertensión y taquicardia subsecuente[8]. 
Myocardial infarction (MeSH), perioperative period (MeSH,

Majr), postoperative period (MeSH, Majr), anesthesia

$$
\text { (MeSH). }
$$

Google Scholar, PubMed-Medline, EMBASE, $n=603$

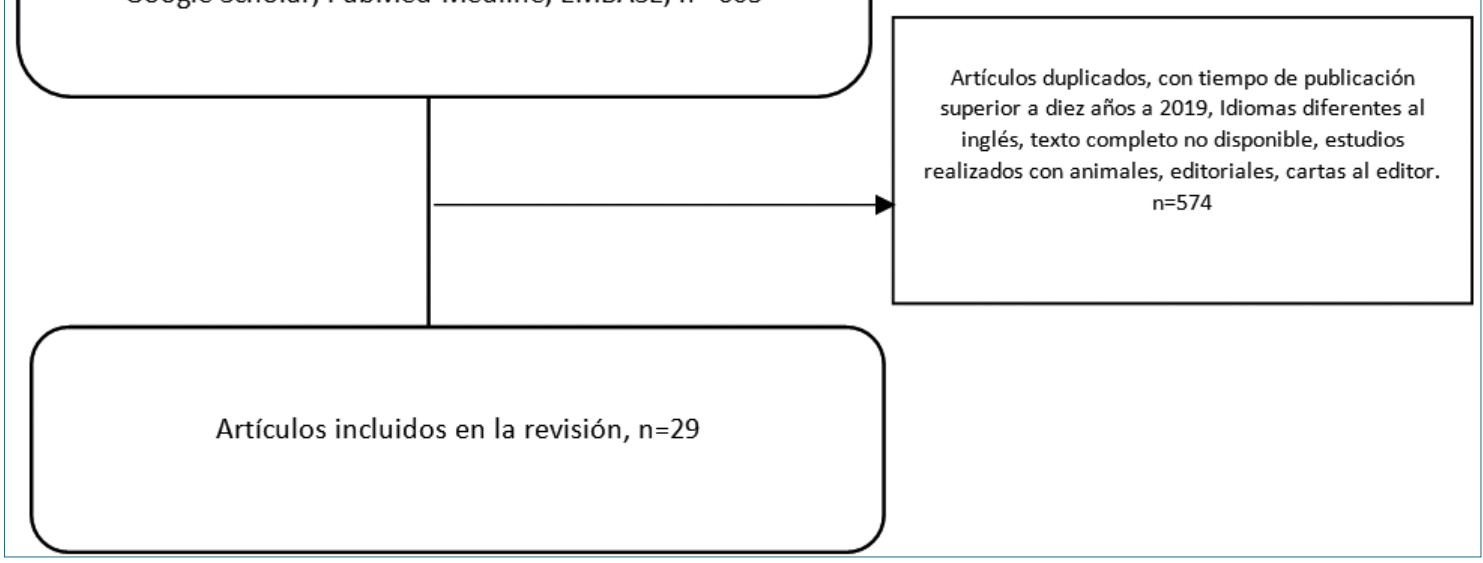

Figura 1.

\section{Manifestaciones clínicas}

La mayoría de los pacientes que sufren IAM perioperatorio se encuentran bajo el efecto residual de anestésicos, por lo que frecuentemente los síntomas suelen enmascararse[9]. Menos del $10 \%$ de los pacientes desarrolla dolor torácico típico, mientras que un 30\% presenta al menos: dolor o un equivalente anginoso (dolor, disnea, síncope), cambio en el EKG o pérdida de la motilidad miocárdica en el ecocardiograma[7]. El estudio POISE, encontró que hasta un $65 \%$ de los pacientes quienes desarrollaron IAM perioperatorio no manifestaron síntomas isquémicos, con aumento del riesgo de mortalidad a 30 días en proporciones similares en aquellos que desarrollaron manifestaciones clínicas y en los que no lo hicieron (OR 4, IC 95\% 2,65-6,06 vs OR 4,76, IC 95\% 2,68$8,43)[10]$.

\section{Factores de riesgo}

Los índices de riesgo cardiovascular validados en el contexto de cirugía no cardíaca como Lee o de Goldman, incorporan los principales factores de riesgo para infarto perioperatorio. Estos se describen más detalladamente en la Tabla 1[11].

Otros factores de riesgo sugeridos en la literatura para IAM son: edad mayor de 70 años, cirugía de aorta abdominal, diabetes mellitus, antecedente de angi- na, alteración prequirúrgica del segmento ST- onda T, artrodesis de rodilla o cadera, la enfermedad arterial periférica, hipotensión clínicamente importante en el transoperatorio, el sangrado mayor, una pobre capacidad (< 4 METS) o clase funcional (III,IV) y un índice de riesgo cardíaco revisado superior o igual a 3 puntos[10]-[12].

\section{Diagnóstico}

Debido al potencial efecto analgésico, sedante y amnésico de los medicamentos comúnmente utilizados en anestesia, es mandatorio realizar estudios complementarios incluyendo electrocardiograma (EKG) de 12 derivaciones y mediciones seriadas de troponinas, a todos aquellos pacientes que refieran dolor anginoso o cualquiera de sus equivalentes, en especial disnea. Asimismo, deben estudiarse los pacientes con inestabilidad hemodinámica de origen no claro y signos de dificultad respiratoria[4].

De acuerdo con la cuarta definición universal, el IAM se define como la elevación de los valores de troponina por encima del percentil 99 del límite superior de referencia, siendo la variación en el tiempo un predictor de agudeza de la lesión miocárdica. Además, de la elevación de los valores de troponina, el diagnóstico de IAM debe estar asociado al menos a alguno de los siguientes: (i) síntomas de isquemia miocárdica; (ii) cambios de la onda T o del segmento 


\begin{tabular}{|c|c|}
\hline & abla 1 \\
\hline Factor de riesgo & Fuente \\
\hline Antecedente de cardiopatía isquémica & Lee, Goldman revisado \\
\hline Antecedente de enfermedad cardiovascular & Lee revisado \\
\hline Insuficiencia cardíaca & Lee revisado \\
\hline Diabetes mellitus dependiente de insulina & Lee revisado \\
\hline $\begin{array}{l}\text { Insuficiencia renal crónica, con creatinina sérica superior } \\
\text { a } 2 \mathrm{mg} / \mathrm{dl}\end{array}$ & Lee, Goldman revisado \\
\hline Edad mayor de 70 años & Goldman revisado \\
\hline Galope S3 o ingurgitación venosa yugular & Goldman revisado \\
\hline Estenosis aórtica severa & Goldman revisado \\
\hline $\begin{array}{l}\text { Ritmo no sinusal o contracciones auriculares prematuras (> } \\
5 \text { en el último EKG) }\end{array}$ & Goldman revisado \\
\hline $\begin{array}{l}\mathrm{PO} 2<60 \mathrm{mmHg}, \mathrm{PCO} 2>50 \mathrm{mmHg}, \mathrm{HCO} 3-<20 \mathrm{mEq} / \mathrm{L}, \mathrm{K} \\
\text { sérico }<3 \text {, signos de insuficiencia renal o hepática crónicas }\end{array}$ & Goldman revisado \\
\hline Cirugía emergente & Goldman revisado \\
\hline Cirugía intraaótica, intraperitoneal o torácica & Goldman revisado \\
\hline
\end{tabular}

ST nuevos o significativos respecto al trazado basal, nuevas ondas Q o bloqueo de novo de rama izquierda en el EKG; (iii) nueva o supuesta evidencia de pérdida de la motilidad segmentaria en el ecocardiograma; (iv) identificación de trombo coronario mediante angiografía o autopsia[4].

Los pacientes con alto riesgo para IAM perioperatorio, deben ser tamizados preoperatoriamente con estudios de troponina, si es posible de alta sensibilidad, en favor de obtener un valor basal que permita identificar la magnitud del cambio. La LMCnC puede considerarse como diagnóstico alterno cuando no se cumplen los criterios para IAM[13].

\section{Tamizaje de la lesión miocárdica perioperatoria}

No existe consenso acerca del cribado de la lesión miocárdica y el infarto perioperatorios. Recomendaciones de expertos, promueven la realización durante las primeras horas del posoperatorio de un estudio de troponina y electrocardiografía, en pacientes con alto riesgo, independientemente de la ausencia de signos y/o síntomas[10].

Aquellos pacientes en quienes se solicita EKG una vez se han obtenido valores elevados de troponina de baja sensibilidad, muestran cambios compatibles con IAM (inversión de onda T vs depresión del segmento ST), entre el 16\%-23\%. Por esta razón, expertos sugieren que los pacientes llevados a cirugía hospitalaria y que tienen factores de riesgo para IAM, deberían ser tamizados rutinariamente mediante troponina en el posoperatorio, en las primeras 6 a 12, así como a las 24, 48 y 72 horas[1],[10].

A la luz de la evidencia actual, no es claro el papel del péptico natriurético cerebral (PNc) en el tamizaje de la lesión miocárdica perioperatoria. Se ha encontrado asociación entre una elevación preoperatoria del biomarcador y peores desenlaces cardiovasculares a 30 días, incluyendo IAM y muerte súbita cardíaca [14]-[17]. En pacientes con medición basal preoperatoria elevada de pro-PNc o PNc, se recomienda la medición rutinaria y seriada de troponina hasta las 48-72 horas del posoperatorio[18].

De otro lado, no existe consenso respecto a la tamización seriada mediante EKG en pacientes sometidos a cirugía no cardíaca. Sin embargo, al menos un estudio electrocardiográfico prequirúrgico de base, debería solicitarse de rutina a todos los pacientes con factores de riesgo para infarto/lesión miocárdica perioperatorios[19],[20].

\section{Pronóstico}

La aparición de lesión miocárdica y el infarto perioperatorios, aumenta considerablemente el riesgo de padecer complicaciones cardiovasculares mayores a 30 días, incluyendo el riesgo de muerte[1],[9],[21]. En este mismo sentido, considerando desenlaces también a 30 días, el IAM perioperatorio aumenta el riesgo de IAM recurrente, paro cardiorrespiratorio no fatal, 
insuficiencia cardíaca cardíaca, reingreso hospitalario y angina progresiva con necesidad de posterior revascularización[10],[22],[23]. Se considera que el IAM perioperatorio aumenta entre $5 \%-25 \%$ el riesgo de muerte a 30 días, según distintas series[1],[24],[25].

A largo plazo, los pacientes que sufren lesión miocárdica tienen una mortalidad a un año mayor que aquellos que no la presentan ( $22,5 \%$ vs $9,3 \%)$. Asimismo, la elevación perioperatoria de troponina 0 CK-MB, incrementa el riesgo de morir a los 48 meses, en comparación con quienes no sufren dicho aumento (OR 2,5, IC 95\% 1,5-4,0)[21].

\section{Manejo}

No existe evidencia suficiente para generar recomendaciones universales acerca del manejo de la lesión/infarto perioperatorios. Un estudio encontró que los pacientes con IAM que reciben aspirina/estatina tienen menor riesgo de mortalidad a 30 días (OR 0,54, IC 95\% 0,29-0,99 y OR 0,26, IC 95\% 0,130,54 , respectivamente), por lo que ambos grupos son tributarios de recibir este tratamiento[10].

Un ensayo clínico aleatorizado, realizado con más de 1.700 pacientes (la quinta parte con diagnóstico de IAM perioperatorio), se encontró que la incidencia de complicaciones cardiovasculares importantes tales como muerte, IAM recurrente no fatal, evento cerebrovascular isquémico (ECVi), enfermedad arterial periférica (EAP) y tromboembolismo venoso (TV); fue menor en el grupo que recibió dabigatrán respecto al que no lo recibió: $11 \%$ vs $15 \%$ (HR 0,72; IC 95\% $0,55-0,93)$. Sin embargo, en el análisis de subgrupos, no hubo diferencia estadísticamente significativa que demostrara la ventaja del uso de dabigatrán para cualquiera de los desenlaces particulares, por lo que, no puede ser recomendado rutinariamente[26]. Por su parte, rivaroxabán podría ser útil si se adiciona a la terapia antiagregante dual en pacientes infartados, o como fármaco coadyuvante a la ASA en pacientes con riesgo cardiovascular alto[27],[28].

En muchas ocasiones, la fibrinólisis no se considera el tratamiento de elección en consideración al riesgo de sangrado. De ser posible, debe considerarse la coronariografía, con o sin colocación de stent. En cualquiera de los dos casos, debe promoverse la antiagregación dual con ASA y un inhibidor del receptor P2Y12 como el clopidogrel (si se ha recurrido a terapia fibrinolítica); o ticagrelor/prasugrel (si se ha realizado intervención percutánea). Los betabloqueadores reducen el trabajo miocárdico y deberían considerarse como coadyuvantes de primera línea en este tipo de pacientes[29]. De cualquier modo, debe vigilarse la posibilidad de adversos como sangrado e hipotensión, deletéreos en el periodo posoperatorio temprano.

\section{Lesión miocárdica sin evidencia de infarto}

Considerando la afectación directa sobre la mortalidad, estos pacientes deben recibir tratamiento inicial con aspirina y estatinas, medicamentos que han mostrado disminuir desenlaces cardiovasculares mayores a 12 meses en esta población. Puede considerarse el inicio de un betabloqueador, pero no existe evidencia de primer nivel para recomendarlo de forma rutinaria[10].

\section{Conclusiones}

La lesión miocárdica y el infarto agudo de miocardio perioperatorios son entidades frecuentes en la práctica anestesiológica. La ausencia de manifestaciones clínicas y el efecto residual de los fármacos anestésicos, usualmente disfrazan el cuadro de forma que puede pasar desapercibido si no se considera el diagnóstico. Su identificación y pronto tratamiento genera un gran impacto sobre la morbimortalidad en términos de disminuir la incidencia de complicaciones cardiovasculares mayores como infarto recurrente no fatal, arritmias y muerte súbita cardíaca tanto a corto como a largo plazo. En este sentido, debe realizarse su búsqueda activa mediante biomarcadores, especialmente en pacientes con riesgo cardiovascular elevado.

\section{Referencias}

1. Botto F, Alonso-Coello P, Chan M, Villar J, Xavier D, Srinathan $\mathrm{S}$, et al. Myocardial injury after noncardiac surgery: a large, international, prospective cohort study establishing diagnostic criteria, characteristics, predictors, and 30-day outcomes. Anesthesiology. 2014;120(3):56478. https://doi.org/10.1097/
ALN.0000000000000113

2. Sheth $T$, Natarajan M, Hsieh V, Valettas N, Rokoss M, Mehta S, et al. Incidence of thrombosis in perioperative and non-operative myocardial infarction. $\mathrm{Br}$ J 
Anaesth. 2018;120(4):725-33.

Doi: 10.1016/j.bja.2017.11.063.

https://doi.org/10.1016/j.

bja.2017.11.063

3. Gualandro D, Campos C, Calderaro D, Yu P, Marques A, Pastana $A$, et al. Coronary plaque rupture in patients with myocardial infarction after noncardiac surgery: frequent and dangerous. Atherosclerosis. 2012;222(1):191-5. Doi: 10.1016/j.atherosclerosis.2012.02.021. https://doi. org/10.1016/j.atherosclerosis.2012.02.021

4. Thygesen K, Alpert J, Jaffe A, Chaitman B, Bax J, Morrow D, et al. Fourth Universal Definition of Myocardial Infarction (2018). Circulation. 2018; 138(20):61851. https://doi.org/10.1161/ CIR.0000000000000617

5. van Waes J, Nathoe $H$, de Graaff J, Kemperman H, de Borst G, Peelen $\mathrm{L}$, et al. Myocardial injury after noncardiac surgery and its association with short-term mortality. Circulation. 2013;127(23):226471. Doi: 10.1161/CIRCULATIONAHA.113.002128. https://doi. org/10.1161/CIRCULATIONAHA. 113.002128

6. Devereaux $P$, Biccard B, Sigamani A, Xavier D, Chan M, Srinathan $S$ et al. Association of Postoperative High-Sensitivity Troponin Levels With Myocardial Injury and 30-Day Mortality Among Patients Undergoing Noncardiac Surgery. JAMA. 2017;317(16):164251. https://doi.org/10.1001/ jama.2017.4360

7. Puelacher $C$, Lurati $G$, Seeberger D, Sazgary L, Marbot S, Lampart A et al. Perioperative Myocardial Injury After Noncardiac Surgery: Incidence, Mortality, and Characterization. Circulation. 2018;137(12):1221-32. https:// doi.org/10.1161/CIRCULATIONAHA. 117.030114

8. Landesberg G, Beattie S, Mosseri M, Jaffe A, Alpert J. Periope- rative myocardial infarction. Circulation 2009;115(22):293644. Doi: 10.1161/CIRCULATIONAHA. 108.828228. https://doi. org/10.1161/CIRCULATIONAHA. 108.828228

9. Devereaux P, Xavier D, Pogue J, Guyatt G, Sigamani A, Garutti I, et al. Characteristics and shortterm prognosis of perioperative myocardial infarction in patients undergoing noncardiac surgery: a cohort study. Ann Intern Med. 2011;154(8):523-8. Doi: 10.7326/0003-4819-154-8201104190-00003. https://doi. org/10.7326/0003-4819-154-8201104190-00003

10. Devereaux $P$, Yang $H$, Yusuf $S$, Guyatt G, Leslie K, Villar J, et al. Effects of extended-release metoprolol succinate in patients undergoing non-cardiac surgery (POISE trial): a randomised controlled trial. Lancet. 2008;371(9627):1839-47. Doi: 10.1016/S0140-6736(08)606017. https://doi.org/10.1016/ S0140-6736(08)60601-7

11. Kaw $R$, Nagarajan $V$, Jaikumar $L$, Halkar M, Mohananey D, Hernández $\mathrm{A}$, et al. Predictive Value of Stress Testing, Revised Cardiac Risk Index, and Functional Status in Patients Undergoing Noncardiac Surgery. J Cardiothorac Vasc Anesth. 2019 Apr;33(4):927932. doi: 10.1053/j. jvca.2018.07.020. https://doi. org/10.1053/j.jvca.2018.07.020

12. Kamel H, Johnston $S$, Kirkham J, Turner C, Kizer J, Devereux $\mathrm{R}$, et al. Association between major perioperative hemorrhage and stroke or Q-wave myocardial infarction. Circulation. 2012;126(2):207-12. https://doi. org/10.1161/CIRCULATIONAHA. 112.094326

13. Beattie W, Karkouti K, Tait G, Steel A, Yip P, McCluskey S, et al. Use of clinically based troponin underestimates the cardiac injury in non-cardiac surgery: a single-centre cohort study in 51,701 consecutive patients. Can J Anaesth. 2012;59(11):1013-22. https://doi.org/10.1007/s12630012-9782-9

14. Karthikeyan G, Moncur R, Levine $O$, Heels-Ansdell $D$, Chan $\mathrm{M}$, Alonso-Coello $\mathrm{P}$, et al. Is a pre-operative brain natriuretic peptide or $\mathrm{N}$-terminal pro-B-type natriuretic peptide measurement an independent predictor of adverse cardiovascular outcomes within 30 days of noncardiac surgery? A systematic review and meta-analysis of observational studies. J Am Coll Cardiol. 2009;54(17):1599606. https://doi.org/10.1016/j. jacc.2009.06.028

15. Lurati Buse G, Koller M, Burkhart C, Seeberger M, Filipovic M. The predictive value of preoperative natriuretic peptide concentrations in adults undergoing surgery: a systematic review and meta-analysis. Anesth Analg. 2011;112(5):101933. https://doi.org/10.1213/ ANE.0b013e31820f286f

16. Rodseth R, Biccard B, Chu R, Lurati Buse G, Thabane L, Bakhai A, et al. Postoperative B-type natriuretic peptide for prediction of major cardiac events in patients undergoing noncardiac surgery: systematic review and individual patient meta-analysis. Anesthesiology. 2013;119(2):27083. https://doi.org/10.1097/ ALN.0b013e31829083f1

17. Rodseth R, Biccard B, Le Manach $Y$, Sessler D, Lurati Buse G, Thabane $L$, et al. The prognostic value of pre-operative and post-operative B-type natriuretic peptides in patients undergoing noncardiac surgery: B-type natriuretic peptide and $\mathrm{N}$-terminal fragment of pro-B-type natriuretic peptide: a systematic review and individual patient data 
meta-analysis. J Am Coll Cardiol. 2014;63(2):170-80. https://doi. org/10.1016/j.jacc.2013.08.1630

18. Duceppe E, Parlow J, MacDonald $\mathrm{P}$, Lyons K, McMullen M, Srinathan $\mathrm{S}$, et al. Canadian Cardiovascular Society Guidelines on Perioperative Cardiac Risk Assessment and Management for Patients Who Undergo Noncardiac Surgery. Can J Cardiol. 2017;33(1):17-32. https://doi. org/10.1016/j.cjca.2016.09.008

19. Fleisher $L$, Fleischmann K, Auerbach A, Barnason S, Beckman J, Bozkurt B, et al. 2014 ACC/ AHA guideline on perioperative cardiovascular evaluation and management of patients undergoing noncardiac surgery: a report of the American College of Cardiology/American Heart Association Task Force on practice guidelines. Circulation. 2014;130(24):221545. https://doi.org/10.1161/ CIR.0000000000000105

20. Kristensen $S$, Knuuti J, Saraste A, Anker S, Bøtker H, Hert S, et al. 2014 ESC/ESA Guidelines on non-cardiac surgery: cardiovascular assessment and management: The Joint Task Force on non-cardiac surgery: cardiovascular assessment and management of the European Society of Cardiology (ESC) and the European Society of Anaesthesiology (ESA). Eur Heart J. 2014;35(35):2383-431. https://doi.org/10.1093/eurhear-

\section{tj/ehu282}

21. Levy M, Heels-Ansdell $D$, Hiralal R, Bhandari M, Guyatt G, Yusuf $S$, et al. Prognostic value of troponin and creatine kinase muscle and brain isoenzyme measurement after noncardiac surgery: a systematic review and meta-analysis. Anesthesiology. 2011;114(4):796806. https://doi.org/10.1097/ ALN.0b013e31820ad503

22. Kim L, Martínez E, Faraday N, Dorman T, Fleisher L, Perler $B$, et al. Cardiac troponin I predicts short-term mortality in vascular surgery patients. Circulation. 2002;106(18):236671. https://doi.org/10.1161/01. CIR.0000036016.52396.BB

23. Smilowitz N, Beckman J, Sherman S, Berger J, Berger J. Hospital Readmission After Perioperative Acute Myocardial Infarction Associated With Noncardiac Surgery. Circulation. 2018;137(22):2332-9. https:// doi.org/10.1161/CIRCULATIONAHA. 117.032086

24. Devereaux P, Mrkobrada M, Sessler D, Leslie K, AlonsoCoello P, Kurz A, et al. Aspirin in patients undergoing noncardiac surgery. N Engl J Med. 2014;370(16):1494-503. https://doi.org/10.1056/NEJMoa1401105

25. Parashar A, Agarwal S, Krishnaswamy A, Sud K, Poddar K, Bassi $M$, et al. Percutaneous Intervention for Myocardial
Infarction After Noncardiac Surgery: Patient Characteristics and Outcomes. J Am Coll Cardiol. 2016;68(4):329-38. https://doi. org/10.1016/j.jacc.2016.03.602

26. Devereaux P, Duceppe E, Guyatt G, Tandon V, Rodseth R, Biccard $B$ et al. Dabigatran in patients with myocardial injury after non-cardiac surgery (MANAGE): an international, randomised, placebo-controlled trial. Lancet 2018; 391(10137):2325-34. https://doi.org/10.1016/S01406736(18)30832-8

27. Mega J, Braunwald E, Wiviott S, Bassand J Bhatt D, Bode C, et al. Rivaroxaban in patients with a recent acute coronary syndrome. N Engl J Med. 2012 Jan 5;366(1):9-19. https://doi. org/10.1056/NEJMoa1112277

28. Eikelboom J, Connolly S, Bosch J, Dagenais G, Hart R, Shestakovska $\mathrm{O}$, et al. Rivaroxaban with or without Aspirin in Stable Cardiovascular Disease. N Engl J Med. 2017 Oct 5;377(14):1319-1330.

29. Amsterdam $E$, Wenger $N$, Brindis R, Casey D, Ganiats T, Holmes D, et al. 2014 AHAVACC Guideline for the Management of Patients with Non-ST-Elevation Acute Coronary Syndromes: a report of the American College of Cardiology/American Heart Association Task Force on Practice Guidelines. J Am Coll Cardiol. 2014 Dec 23;64(24):e139e228. https://doi.org/10.1016/j. jacc.2014.09.017 